\title{
Construimos un calentador de agua solar para trabajar la sostenibilidad
}

\author{
María Begoña Carretero Gómez \\ IES Valle de Lecrin (Dúrcal) Granada. España.begocarretero@hotmail.com
}

[Recibido en julio de 2011, aceptado en diciembre de 2011]

Estamos inmersos en plena Década de la Educación para la Sostenibilidad. Si queremos impulsar actitudes orientadas al cuidado del medioambiente y su sostenibilidad es necesario trabajar desde los centros de enseñanza e introducir a nuestro alumnado en los problemas medioambientales y sus posibles soluciones. Por ello, en nuestro centro, hemos pensado que una forma de acercarles esta problemática sería planificando actividades que, estando relacionadas con la vida cotidiana, les permitieran ser conscientes de la situación actual. Por ello hemos puesto en marcha la construcción de un calentador de agua solar, con la esperanza de que tanto ellos como sus familias tomen conciencia de la realidad y actúen colaborando en la reducción de la emisión de gases efecto invernadero.

Palabras clave: educación para la Sostenibilidad; calentador de agua solar; cambio climático; energías alternativas

\section{To built a solar hot water heater to work the sustainability problem}

We are commemorating the Education Decade for Sustainable Development. If we want to create positive towards our environment and its sustainable development we have to begin working at school. It is necessary to show our students what problems of the environment are and which solutions can be adopted. That is the reason we have planned this activity in our secondary school. We do think that by doing daily activities we have a good opportunity to fulfil this goal. An example of such experiences is the fabrication of a solar hot water heater to make them and their families more environment conscience.

Keywords: education for Sustainability; solar hot water heater; climate change; alternative energies.

\section{Justificación: ¿por qué esta actividad y por qué ahora?}

Nuestro mundo actual se enfrenta a grandes problemas medioambientales, hoy en día hay que tener en cuenta que entre los 20 países más ricos del mundo han consumido más materia prima y energías no renovables que toda la humanidad a lo largo de su historia y prehistoria, además de generar las dos terceras partes de las emisiones de $\mathrm{CO}_{2}$ (Vilches y Gil, 2003). Hoy en día es necesario fomentar el desarrollo de tecnologías que estén orientadas a cubrir las necesidades básicas a la vez que contribuyen a reducir las desigualdades (Vilchez y Gil Pérez, 2003). Es técnicamente factible reestructurar el sistema energético para que, a la vez que se cumplen los objetivos ambientales, se cubran las necesidades energéticas al 100\% mediante el uso de energías renovables (Educadores para la Sostenibilidad, 2006).

Como se pone de manifiesto en algunos informes, entre los que destacamos GEO-4 Perspectivas del Medio Ambiente Mundial, el Programa de las Naciones Unidas para el Medio Ambiente (2008) y el Informe sobre Desarrollo Humano 2007-2008, el planeta Tierra se encuentra en una situación crítica e insostenible. De esta situación somos todos responsables de una manera u otra, aunque no todos hemos colaborado por igual, (no todos los países contaminamos, ni eliminamos residuos de la misma manera). Es de vital importancia luchar para reducir las emisiones de gases de efecto invernadero si queremos combatir el cambio climático y sus graves consecuencias (Hartman, 2011). En este proceso debemos colaborar todos dentro de nuestras posibilidades y es por ello que la Asamblea General de las Naciones Unidas declaró el periodo comprendido entre 2005 y 2014 como la Década de la Educación 
para la Sostenibilidad, durante el cual se nos anima a los educadores a contribuir proporcionando a nuestro alumnado una percepción lo más cercana posible de los problemas medioambientales a los que hoy en día se enfrenta la humanidad: calentamiento global, contaminación, escasez de combustibles fósiles, pérdida de biodiversidad, etc. Para llamar la atención sobre la gravedad de los riesgos relacionados con la acción humana sobre el planeta y con la idea de resaltar la necesidad y la urgencia de tomar decisiones orientadas a frenar las numerosas actuaciones que se están produciendo, Bybee (1991) utiliza la expresión "emergencia planetaria".

Necesitamos poner freno a este problema ya que, si no, nuestro futuro y el del planeta corren serio peligro. Si el avance hacia sociedades sostenibles exige, como señalan expertos de diferentes campos, una revolución del pensamiento, una nueva forma de entender la relación de los seres humanos con la naturaleza, un nuevo modelo de producción y consumo, nuevos estilos de vida... entonces, las medidas educativas, aunque no las únicas, juegan un papel crucial en este proceso (Posada y Barandiarán, 2010). Se hace necesario actuar de forma simultánea en varios frentes a la vez. Se están dando pasos hacia un sostenibilidad, pero muchos siguen actuando como si el medio pudiera soportarlo todo. Desde la educación para la sostenibilidad es necesario contribuir a despertar la conciencia de la ciudadanía para que se haga cargo de la situación y pase a la acción (Prieto y España, 2010).El actual modelo socioeconómico de los países desarrollados, es insostenible pues es el responsable de numerosos problemas medioambientales que amenazan al planeta, por lo que es necesario un nuevo modelo de sociedad que haga posible un futuro más sostenible, en lo que la educación juega un papel fundamental (Novo, 2006; Vega Marcote, Freitas, Álvarez y Fleuri, 2007; Álvarez y Vega 2009.

Hemos elegido gastar en un corto plazo los numerosos y suficientes recursos que posee nuestro planeta. Se supone que así aumentan nuestros beneficios porque se eleva la renta per capita, la producción, el consumo, la actividad, etc. El problema es que los elementos clave del capital natural: la tierra cultivable, las aguas subterráneas, los bosques, los bancos de pesca, el petróleo, etc. son finitos y no siguen ese patrón de incremento del capital (Prieto y España, 2010). Hay que buscar soluciones que no pueden ser soluciones puntuales ni aisladas ya que estamos hablando de problemas a la vez locales y globales, así que se requiere un entramado de medidas que se apoyen mutuamente (Gil y Vilches, 2006).

Ya en 1992 los gobiernos reunidos en la llamada Cumbre de Río acordaron un plan de acción denominado Agenda 21. En él podemos comprobar como varios de sus capítulos hacen mención a la gran importancia que tiene la educación si queremos promover la sostenibilidad y mejorar la capacidad de la población de afrontar los problemas medioambientales, ya que con la educación podemos cambiar actitudes, fomentar el espíritu crítico y de decisión de los individuos (Novo, 2006; Álvarez y Vega, 2009; Prieto y España, 2010). Los educadores debemos conocer y dar a conocer en las aulas esta situación, incorporando la problemática de la sostenibilidad a nuestras actividades para contribuir a formar una ciudadanía consciente de los riesgos y preparada para tomar decisiones (España y Prieto, 2009; Vilches y Gil, 2008).

Por lo general el acceso a la información que tiene la mayor parte de la población, se reduce a los medios de comunicación de masas, los cuales suelen tratar el tema de forma inconexa y sin gran profundidad (Vilches y Gil, 2009). Es por ello que nosotros como educadores, tenemos la responsabilidad de formar, informar y ayudar a la alfabetización científica de nuestro alumnado, fomentando en ellos actitudes, comportamientos y formas de pensar que colaboren en la consecución de un futuro sostenible. Esta es la razón por la que, en el IES Isabel la Católica de Guadahortuna, hemos considerado que este es un buen momento para empezar a 
trabajar y hacer que los adolescentes de hoy se conviertan en adultos responsables el día de mañana poniendo nuestro pequeño grano de arena con el desarrollo de esta experiencia.

Son muchos los que piensan que con cambios aislados en los modelos de comportamiento en los que modificamos un poco nuestras costumbres y estilos de vida, no vamos a conseguir nada, consideran estas acciones como irrelevantes. Sin embargo encontramos que los estudios realizados nos demuestran justo lo contrario (Vilches et al., 2008; Educadores para la Sostenibilidad, 2008), que la realidad es muy distinta, ya que pequeñas reducciones en el consumo energético por persona, pueden dar lugar a un ahorro considerable de energía y la consiguiente reducción de la contaminación, a la vez que implicamos a la ciudadanía en la construcción de un futuro sostenible.

Creemos que una parte de la población está dispuesta a colaborar en la medida de sus posibilidades intentando paliar este daño de la mejor manera posible. Una forma de colaborar sería fomentando un uso inteligente de las energías alternativas pero en realidad hacemos poco uso de ellas, en general la población las considera como algo lejos de su alcance. Como ya hemos indicado anteriormente, nosotros los educadores, tenemos la posibilidad de formar a las generaciones futuras desarrollando en ellos un espíritu solidario y comprometido con la sociedad en la que viven y el medio ambiente que les rodea. Como en el departamento de Ciencias Naturales de nuestro IES estamos convencidos de ello, ya en el curso 2009 estuvimos trabajando con el diseño y construcción de cocinas solares (Carretero, 2010) así que hemos pensado que siguiendo la misma línea, y para completar el trabajo sobre energías alternativas (energía solar) sería adecuada la experiencia de construir un calentador solar. De igual manera, lo mismo que en el caso de la cocina solar, pensamos que la asignatura de Ámbito Científico Tecnológico de $3^{\circ}$ de la ESO sería la adecuada para poner en marcha este trabajo por varias razones: es un grupo pequeño con tan solo 7 alumnos; en ella podemos integrar con facilidad contenidos de este tipo relacionando medio ambiente, tecnología, sociedad; la carga lectiva es mucho mayor que en otras asignaturas lo cual, entre otras razones, nos ha facilitado mucho el trabajo.

\section{Objetivos: ¿qué pretendemos conseguir con esta experiencia?}

Con esta actividad hemos intentado alcanzar y trabajar objetivos de muy distinta índole. Es un trabajo con el que, a grandes rasgos, podemos tratar objetivos y contenidos científicos, tecnológicos, medioambientales a la vez que desarrollamos en el alumnado un espíritu solidario, introduciéndolos en el problema de la sostenibilidad del medio ambiente.

Concretamente estos han sido los siguientes:

i.Explicar y dar a conocer en que consiste la Década de la Educación para la Sostenibilidad Conocer la problemática energética global y sus relaciones con la actividad humana, desarrollando la capacidad de analizar conflictos socioambientales.

ii.Iniciar en el alumnado un cambio de actitud que les ayude a desarrollar su capacidad para actuar a la vez que utilizan los conocimientos adquiridos para buscar soluciones a los problemas medioambientales.

iii.Ser conscientes de la utilidad de las energías alternativas, en nuestro caso el Sol.

iv.Promover en el alumnado un estilo de vida sostenible a través de la conservación y respeto al medio ambiente, desarrollando en ellos una actitud solidaria entre los propios compañeros y hacia el medio.

v.Fomentar el debate entre las distintas posturas y desarrollar en ellos la capacidad de llegar a acuerdos. 
vi.Aprender a debatir diferentes alternativas y tomar decisiones tanto individuales como colectivas.

vii.Trabajar en grupo de una forma colaborativa y participativa.

viii.Desarrollar en el alumnado destrezas en el uso de las nuevas tecnologías de la información con la búsqueda de datos así como en la organización de los mismos.

ix.Desarrollar destrezas manuales con la fabricación del calentador solar (realización de medidas, manejo de proporciones, utilización de diferentes herramientas, etc.).

x.Aprender a trabajar con materiales cotidianos a partir de los cuales poder construir mecanismos (calentador) útiles.

xi.Desarrollar en ellos el hábito de las tres "R".

xii.Hacer que nuestro alumnado actúe de ante sus propias familias y entorno más cercano como "embajador" y transmisor de información de manera que el espíritu de conservación y cuidado del medio ambiente se extienda en su comunidad.

xiii.Acercar el uso de la energía solar a nuestra comunidad educativa demostrando lo fácil y asequible que es.

xiv.Relacionar contenidos de diferentes materias con el día a día, haciendo de esta forma más atractivas y cercanas aquellas áreas del currículo que puedan resultar más áridas para nuestro alumnado.

La tabla 1 muestra la relación entre los objetivos planteados y las competencias básicas que se pretenden desarrollar.

\begin{tabular}{|c|c|}
\hline \multicolumn{2}{|c|}{ RELACION ENTRE LOS OBJETIVOS PLANTEADOS Y LAS COMPETENCIAS BÁSICAS } \\
\hline \hline Comunicación lingüística & $1,6,13$ \\
\hline \hline Matemática & 10, \\
\hline \hline Conocimiento e interacción mundo físico & $1,2,3,4,5,12,13,14$ \\
\hline \hline Tratamiento información y c. digital & 9 \\
\hline \hline Social y ciudadana & $1,2,3,5,6,7,8,12,13,14$ \\
\hline \hline Cultural y artística & 13 \\
\hline \hline Aprender a aprender & $3,11,15$ \\
\hline \hline Autonomía e iniciativa personal & 10,11, \\
\hline
\end{tabular}

Tabla 1. Objetivos y competencias básicas.

\section{Material y métodos: ¿cómo hemos realizado la experiencia?}

La idea de esta experiencia era el poder demostrar al alumnado como, es relativamente fácil, poder aprovechar los recursos renovables que la naturaleza pone a nuestra disposición de una forma sencilla y sin necesidad de grandes instalaciones. Como el trabajo de construcción del horno solar había dado tan buenos resultados, tomamos este nuevo reto con gran determinación y con la esperanza de, si no superar el trabajo previo, al menos si igualarlo de 
manera que pusimos todos un gran entusiasmo en ello. Nuestro plan de trabajo ha sido el siguiente:

- Buscamos información relacionada con las diferentes formas de aprovechar la energía solar, así como de sus características. Entre otras muchas, podemos destacar aquellas dirigidas a la construcción de cocinas solares parabólicas, hornos solares, colectores planos, calentadores solares, etc (Fundación Terra, 2002; Ayats, 2006; Solar cookers internacional, 2004).

- Demostración del efecto invernadero de una manera sencilla utilizando materiales de fácil acceso como son botellas de plástico, tinta y termómetros. Para ello nos hemos basado en la información obtenida de la página de Greenpeace. ${ }^{1}$

- Hemos representado gráficas a partir de las tablas elaboradas con los datos obtenidos de las medidas de las variaciones de temperatura en función del tiempo.

- Recopilación de información sobre diferentes modelos y formas de construcción de calentadores solares. Valorando los puntos positivos y negativos de cada uno de ellos y decidiendo cual era el modelo más adecuado a nuestras posibilidades (Tabla 2).

- Recogida de material. Todo ha sido bien reciclado (latas, caja de cartón, cristal...) bien aprovechando material sobrante de otras prácticas (pintura), o bien usando material propio del centro (la manguera, tubo de riego por goteo, o el bidón). De manera que el gasto económico ha sido nulo, con lo cual hemos demostrado al alumnado que no es necesario el consumo por el consumo, si no que podemos aprovechar muchas de las cosas que hay en nuestro entorno.

- Toma de datos de la temperatura alcanzada en el calentador que quedaron recogidos en su correspondiente tabla y representados gráficamente.

\begin{tabular}{|c|c|c|}
\hline \multicolumn{3}{|c|}{ Tabla comparativa entre diferentes modelos de calentadores } \\
\hline Modelo & Ventajas & Inconvenientes \\
\hline $\begin{array}{l}\text { Con latas de } \\
\text { refrescos }\end{array}$ & $\begin{array}{ll}\sqrt{ } & \text { Material fácil de obtener } \\
\sqrt{ } & \text { Homogeneidad de tamaño de las } \\
& \text { latas } \\
\sqrt{ } & \text { Poco pesado } \\
\end{array}$ & $\begin{array}{ll}\sqrt{ } & \text { Posibilidad de escape de agua en las } \\
& \text { juntas del tubo. } \\
\sqrt{ } & \text { Hacer los orificios en las latas }\end{array}$ \\
\hline $\begin{array}{l}\text { Con botellas de } \\
\text { PET }\end{array}$ & $\begin{array}{ll}\sqrt{ } & \text { Más facilidad de perforar } \\
\sqrt{ } & \text { Material ligero }\end{array}$ & $\begin{array}{ll}\sqrt{ } & \text { Una mayor variedad de tamaños y } \\
& \text { formas } \\
\sqrt{ } & \text { Posibilidad de escape de agua en las } \\
& \text { juntas del tubo. } \\
\sqrt{ } & \text { Hacer los orificios en las botellas }\end{array}$ \\
\hline $\begin{array}{l}\text { Con botellas de } \\
\text { vidrio }\end{array}$ & $\sqrt{ }$ Más higiénico & $\begin{array}{ll}\sqrt{ } & \text { Más pesado } \\
\sqrt{ } & \text { Más frágil } \\
\sqrt{ } & \text { Menos seguro } \\
\sqrt{ } & \text { Más difícil de perforar }\end{array}$ \\
\hline Colector plano & $\sqrt{ } \quad$ Fácil manejo & $\sqrt{ }$ Menos efectivo \\
\hline
\end{tabular}

Tabla 2. Comparativa entre diferentes modelos de calentadores.

Cuando realizamos la búsqueda de los diferentes modelos de calentadores solares no encontramos una gran variedad de ellos (al menos no tanta como hornos solares) los había hechos con botellas de vidrio, con botellas de refresco, pero nos pareció más fácil el que utilizaba latas de bebidas refrescantes, así que adaptamos la información encontrada para

\footnotetext{
${ }^{1}$ http://www.solarizate.org/pdf/castellano/laboratorio/A_calor_de_.pdf
} 
fabricar un sistema de calefacción y hacer nuestro propio calentador de agua. Son muchas las direcciones de Internet encontradas pero nosotros elegimos fabricar el nuestro usando latas de refresco. ${ }^{2}$

\section{Conclusiones}

Ha sido una actividad de gran muy fructífera no solo por haber despertado en nuestros chicos y chicas un espíritu solidario, y protector del medio ambiente sino por habernos permitido a la vez, desarrollar conocimientos y destrezas propios de diferentes áreas (matemáticas, ciencias sociales, tecnología, física...) encontrando un punto de convergencia entre todas ellas.

A la vez hemos procurado que hubiera una interconexión entre la vida cotidiana y las ciencias. El tema elegido está muy próximo a su actividad diaria, lo que ha servido para reforzar más su implicación y motivación por el aprendizaje a la vez que desarrollaban su actitud a favor del medio ambiente. Ha sido una forma diferente de aprender, muy participativa y permitiendo que nuestro alumnado interactúe de manera constante. No solo los que han sido encargados de de llevar a cabo la actividad, sino también del resto de la comunidad educativa. A todos les ha llamado la atención y han mostrado su interés, han sido bastantes los grupos de alumnos que se han acercado de manera curiosa haciendo preguntas sobre nuestro calentador, todos han querido colaborar de una manera u otra. Estamos de acuerdo con Bendala y Pérez (2004) cuando dicen que "este tipo de interacción ciencias-vida cotidiana hace que el aprendizaje sea más significativo para el alumnado".

Por otro lado hemos reforzado la idea de la gran utilidad de las energías alternativas, a al vez que su actitud hacia ellas ha cambiado puesto que ya no les parece algo fuera de su alcance. Esto nos ha llevado a pensar que con este tipo de actividades la educación adquiere un papel fundamental en ayudar a la construcción de sociedades y futuros más sostenibles. Fomentando acciones individuales y colectivas que ayudan a cambiar hábitos, comportamientos y actitudes por otros más sostenibles.

Con esta experiencia hemos hecho hincapié en el desarrollo de valores de una forma más atractiva a nuestro alumnado ya que como bien indican Ojeda y Perales (2008) "con actividades de este tipo se contribuye a su desarrollo cognitivo, afectivo, social y moral a la vez que se favorecen hábitos de discusión, crítica e investigación fuera del esquema tradicional". Hemos promovido en ellos actitudes y comportamientos que contribuyen a la sostenibilidad al tiempo que hemos aumentado el interés por la materia, puesto que hemos conectado esta experiencia con una problemática real. Todo ello favorece un mejor aprendizaje y adquisición de habilidades que le permitan poder seguir aprendiendo, a la vez que colaboran en el desarrollo sostenible (Escudero, Cid y Escudero, 2009). Se trata de formar ciudadanos y ciudadanas conscientes de los problemas y que a la vez estén preparados para asumir las decisiones adecuadas (Gil y Vilchez, 2006).

Se hace necesario educar a la población el uso de energías renovables y que mejor forma de empezar que en los centros escolares y de una forma tan amena y enriquecedora como esta.

Por último y en relación con el proceso de construcción de nuestro calentador hemos de añadir una propuesta de mejora a tener en cuenta en situaciones futuras. El ensamblaje de los tubos ha sido muy laborioso, en ocasiones ha sido necesario calentar un poco el tubo para que encajase bien en las piezas de unión. Como el número de uniones ha sido muy elevado, cuando hemos hecho circular el agua por él se han producido pequeñas fugas de agua. Como consecuencia, hemos creído que se podría sustituir el tubo por una simple goma de regar

\footnotetext{
${ }^{2}$ http://www.gstriatum.com/energiasolar/blog/2009/04/22/calentador-solar-con-latas-de-refresco-o-cerveza/
} 
pintada de negro, lo que además de proporcionarnos un material más flexible, evitaría las fugas al ser innecesario hacer tantos empalmes.

\section{Referencias}

Álvarez, P. y Vega, P. (2009). Una propuesta educativa para la sostenibilidad. En R. García Mira y P. Vega Marcote (Directores), Sostenibilidad, valores y cultura ambiental (pp.84104). Madrid: Ediciones Pirámide.

Ayats, M, 2006. La paracocina documento en línea: http://solarcooking.org/espanol /paracuina-es.pdf

Bendala, M.; Ortega J.A. (2004). Educación ambiental: praxis científica y vida cotidiana. Descripción de un proyecto. Revista Eureka sobre Enseñanza y Divulgación de las Ciencias, 1 (3), 233-239. En línea en: http:// reuredc.uca.es

Bybee, R. W. (1991). Planet earth in crisis: how should science educators respond? American Biological Teacher, 53(3), 146-153.

Carretero, M.B. (2010). El sol, la cocina solar y la solidaridad: una receta muy sabrosa. Revista Eureka sobre Enseñanza y Divulgación de las Ciencias, 7(2), 544-557. En línea en: http://reuredc.uca.es.

Educadores para la Sostenibilidad. (2006). Tecnologías para la sostenibilidad. Revista Eureka sobre Enseñanza y Divulgación de las Ciencias, 3(3), 517-519. En línea en: http://reuredc.uca.es

Educadores para la Sostenibilidad. (2008). 2008, año internacional del planeta Tierra. Una nueva iniciativa convergente con los objetivos de la década de la educación por un futuro sostenible. Revista Eureka sobre Enseñanza y Divulgación de las Ciencias, 5(2), 235 236. En línea en: http://reuredc.uca.es.

Escudero, M.; Cid C. y Escudero, R. (2009). Controversia de los agrocombustibles, una propuesta didáctica para las ciencias del mundo contemporáneo. Revista Eureka sobre Enseñanza y Divulgación de las Ciencias, 6(1), 131-139. En línea en: http:// reuredc.uca.es.

España, E. y Prieto, T. (2009) Educar para la sostenibilidad: el contexto de los Problemas socio-científicos. Revista Eureka sobre Enseñanza y Divulgación de las Ciencias, 6(3), 345354. En línea en: http://reuredc.uca.es.

Fundación Terra (2002). Cocina solar. Suplemento perspectiva ambiental No 25

GEO-4 Perspectivas del Medio Ambiente Mundial. Programa de las Naciones Unidas para el Medio Ambiente. Madrid: Mundi-Prensa. (UNEP) (2008)

Gil, D. y Vilches, A. (2006). Algunos obstáculos e incomprensiones en torno a la sostenibilidad. Revista Eureka sobre Enseñanza y Divulgación de las Ciencias, 3(3), 507-516. En línea en: http:// reuredc.uca.es.

Hartmann, T. (2011). Las últimas horas de la vieja luz del sol. Barcelona: Icaria. Menéndez, R y Moliner, R (Coords.). (2011). Energía sin CO2. Madrid: CSIC/CATARATA.

Informe sobre Desarrollo Humano 2007-2008. Programa de las Naciones Unidas para el desarrollo (2008). Madrid: Mundi-Prensa.

Novo, M. (2006). El desarrollo sostenible. Su dimensión ambiental y educativa. Madrid: Pearson Educación 
Ojeda Barceló, F. y Perales, F. J. (2008). Ecourban: Nuevos caminos para nuevas ideas en educación ambiental. Revista Eureka sobre Enseñanza y Divulgación de las Ciencias, 5(1), 7593

Posada, R. y Barandiarán, J. (2010). Educando para un futuro sostenible: una aportación desde las clases de ciencias de la ESO. Revista Eureka sobre Enseñanza y Divulgación de las Ciencias, 7 número extraordinario 2010, 316-329. En línea en: http:/ / reuredc.uca.es.

Prieto, T. y España, E. (2010). Reflexiones teóricas educar para la sostenibilidad. Un problema del que podemos hacernos cargo. Revista Eureka sobre Enseñanza y Divulgación de las Ciencias, 7, número extraordinario 2010, 216-229. En línea en: http://reuredc.uca.es.

Solar nternacional (2004). Cocinas solares: como construirlas y utilizarlas. En linea: http://solaina.es/drupal/files/cocinas_solares_como_construirlas_y_utilizarlas.pdf

Vega Marcote, P.; Freitas, M.; 8oscazo Suárez, P.; Fleuri, R. (2007) Marco teórico y metodológico de educación ambiental e intercultural para un desarrollo sostenible. Revista Eureka sobre Enseñanza y Divulgación de las Ciencias, 4(3), 539-554. En línea en: http://reuredc.uca.es.

Vilches, A. y Gil, D. (2003). Construyamos un futuro sostenible. Diálogos de supervivencia. Madrid: Cambridge University Press.

Vilches, A. y Gil, D. (2008). La construcción de un futuro sostenible en un planeta en riesgo. Alambique, 55, 9-18.

Vilches, A. y Gil, D. (2009). Una situación de emergencia planetaria, a la que debemos y "podemos" hacer frente. Revista de Educación, número extraordinario 2009, 101-122. 


\section{Anexo.- Proceso de fabricación}

Los materiales utilizados han sido:

Latas de refresco de $33 \mathrm{cl}$

- Tubo de riego por goteo, codos y piezas en "T" para hacer la instalación del agua.

- Pintura negra

- Caja de cartón

- Corcho blanco

- Cristal de metacrilato

- Manguera

- Depósito o bidón para el agua.

El procedimiento seguido constó de los siguientes pasos:

$>$ Se hicieron agujeros en las latas con el diámetro adecuado para que pudiera pasar el tubo por su interior (figuras 1 y 2).

$>$ Posteriormente se pintaron todas de color negro (figura 3).

> Fabricamos el circuito de tubos por donde circularía el agua (figuras 4 y 5).

> Una vez montado preparamos la caja, para ello la forramos con corcho blanco (figura 6) que se encargaría de aislar el interior y evitar pérdidas de calor, y lo pintamos todo de negro.

> Por último lo motamos todo y pusimos el cristal que quedo sellado (figura 8).

$>$ El circuito (figura 7) quedó terminado cuando lo conectamos al bidón donde se almacenaría el agua.

> El calentador quedó dispuesto en el patio del instituto en una zona orientada al sur y con una inclinación de aproximadamente $45^{\circ}$ (figura1).

Lo único que nos quedaba era probarlo. Para ello colocamos un termómetro con la finalidad de saber que temperatura se alcanzaba. Nos quedamos sorprendidos, llegó a marcar $65^{\circ} \mathrm{C}$ en tan solo unos minutos (figura 10).

\begin{tabular}{|l|l|}
\hline \multicolumn{2}{|c|}{ Proceso de fabricación } \\
\hline Lavar la latas & \\
\hline Perforar las latas con una barrena & Figura 1 y 2 \\
\hline Pintar de negro & Figura 3 \\
\hline Construir del circuito con tubo de plástico & Figuras 4 y 5 \\
\hline Forrar la caja con paneles de corcho blanco & Figura 6 \\
\hline Pintar de negro toda la caja & \\
\hline Montar el circuito dentro de la caja & Figura 7 \\
\hline Poner el cristal y sellar & Figura 8 \\
\hline Conectar con el bidón de agua & Figura 9 \\
\hline Medir la temperatura & Figura 10 \\
\hline
\end{tabular}




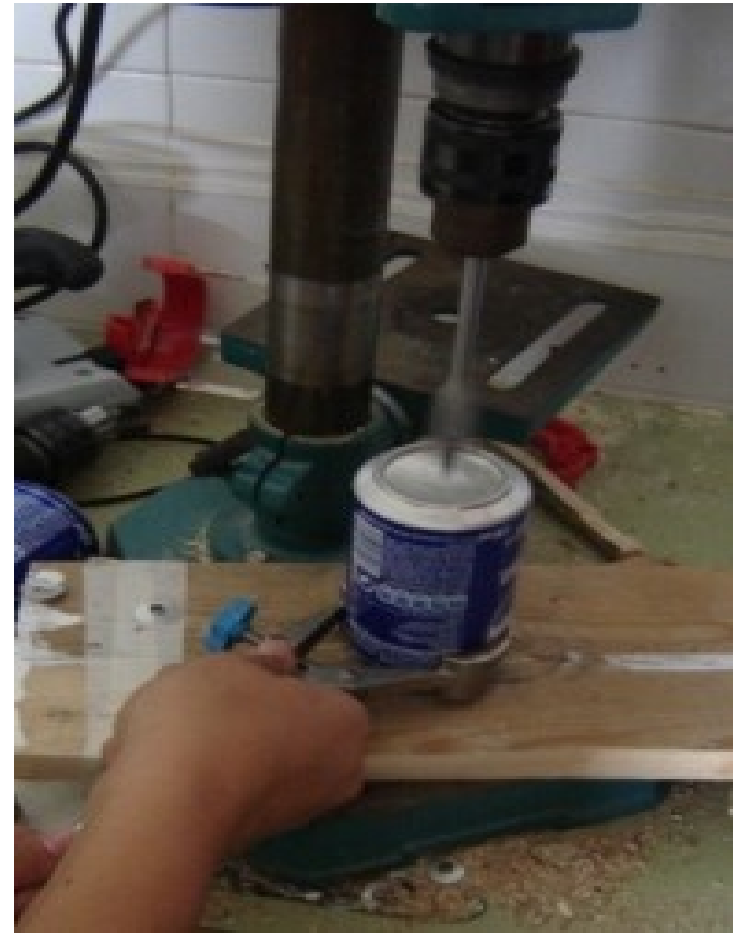

Figura 1

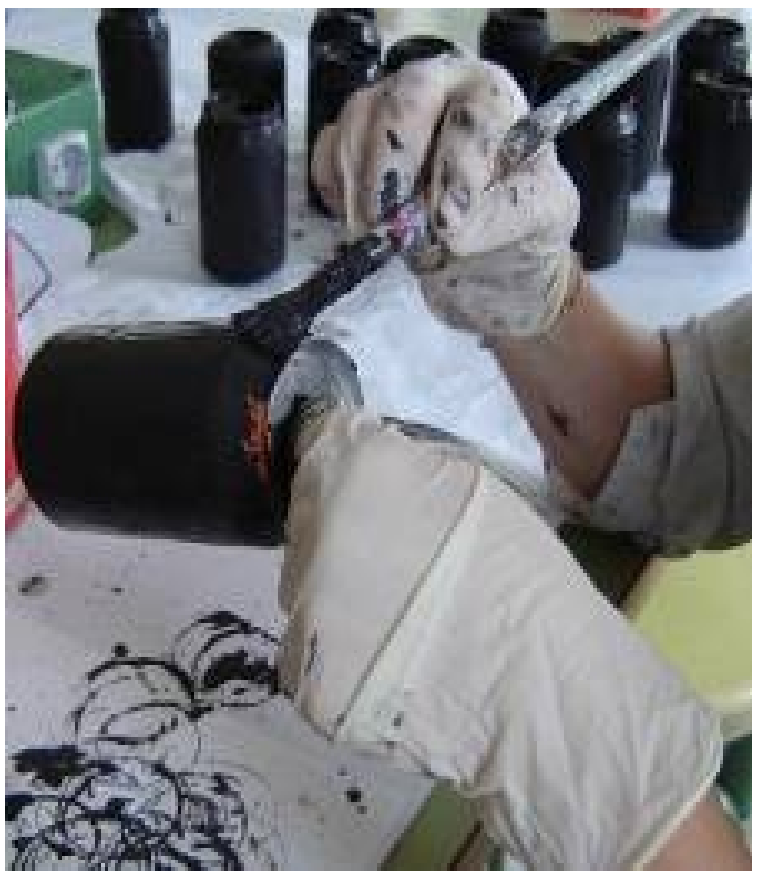

Figura 3

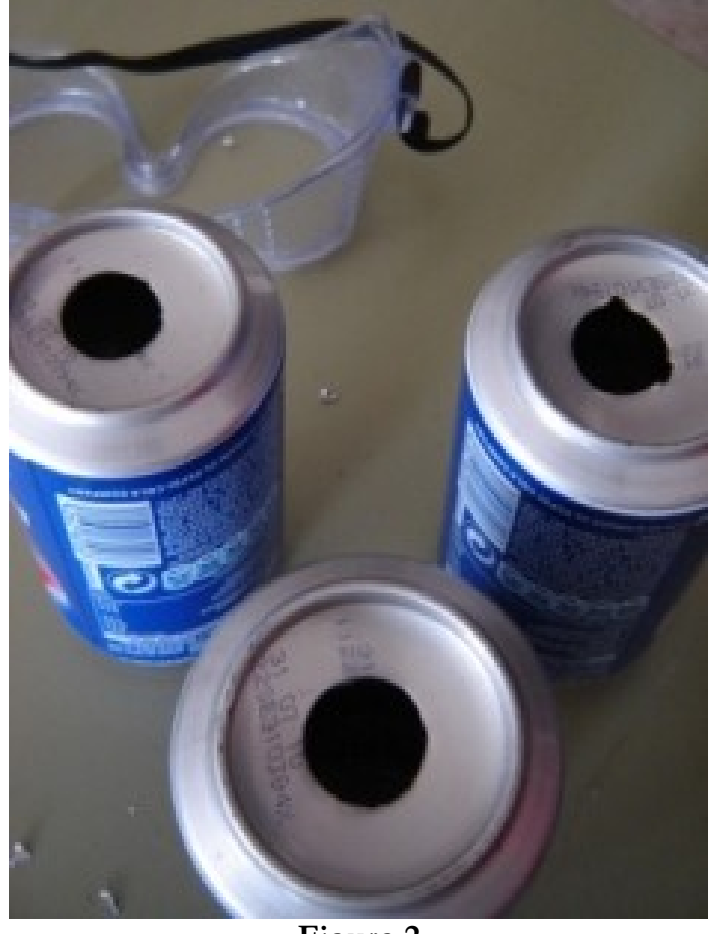

Figura 2

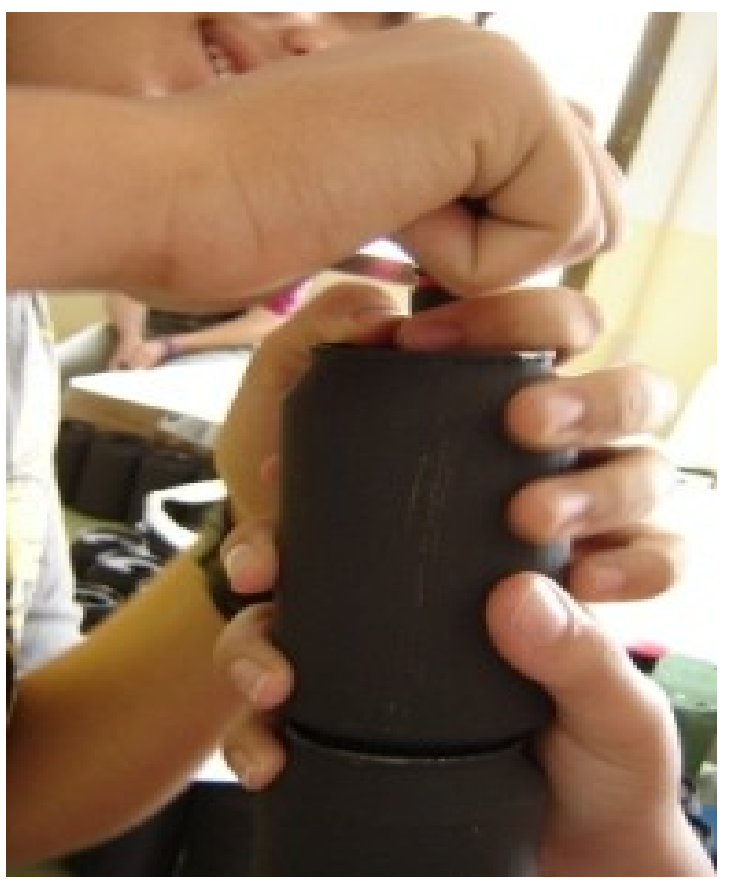

Figura 4 


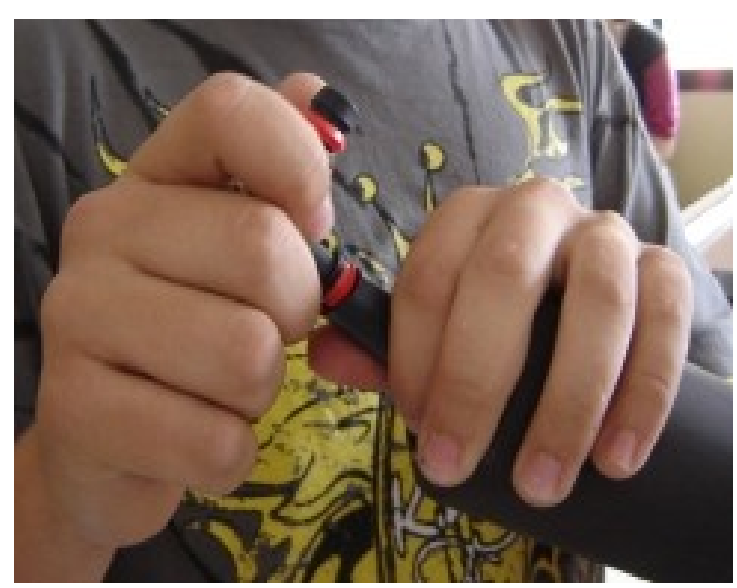

Figura 5

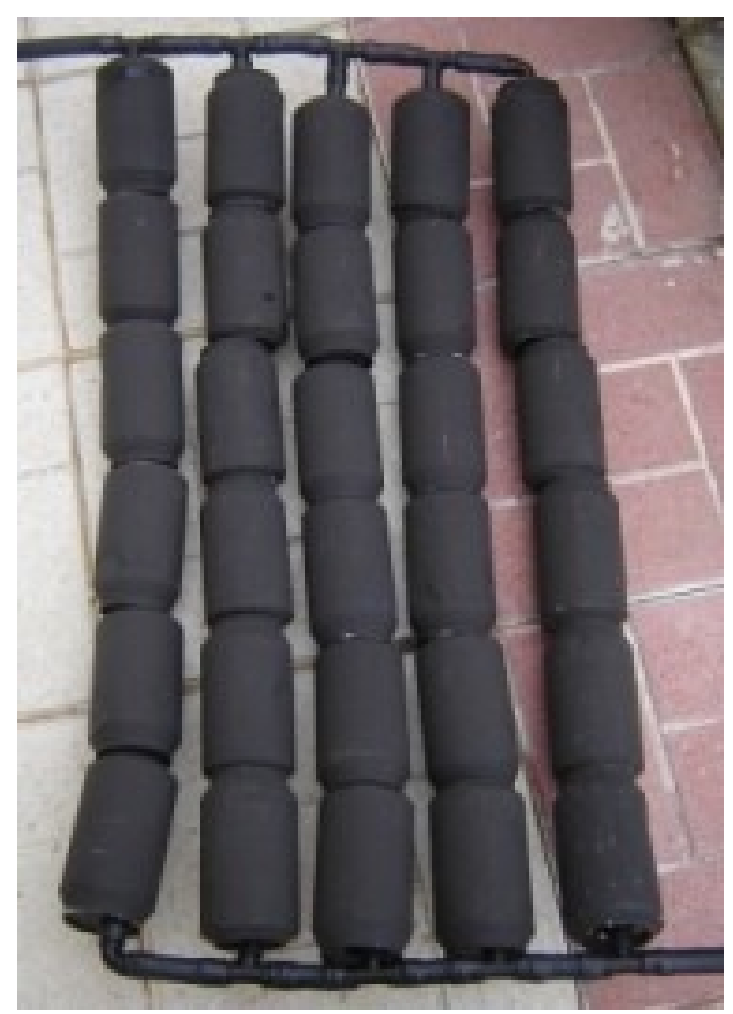

Figura 7

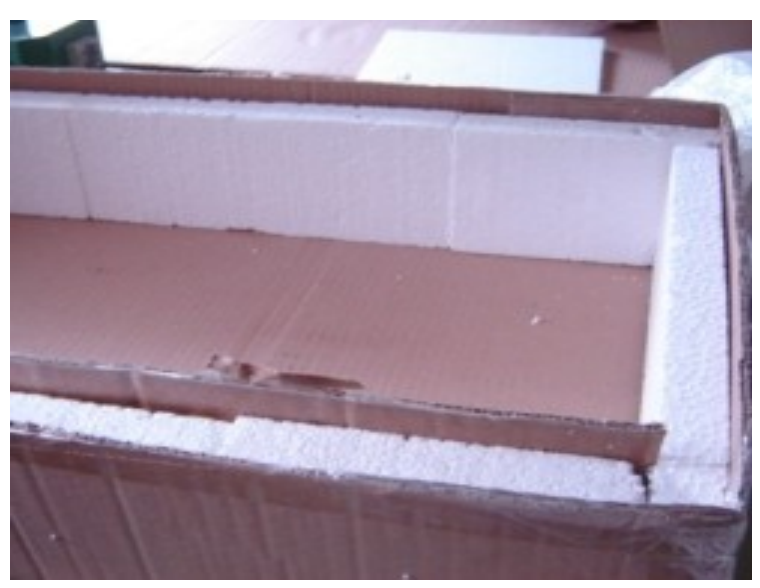

Figura 6

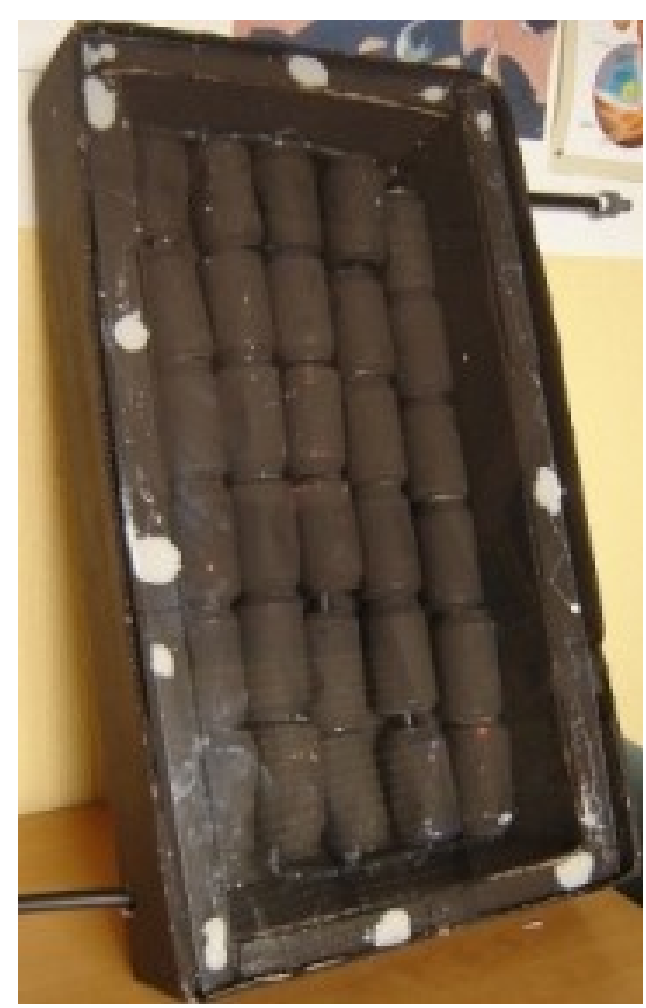

Figura 8 


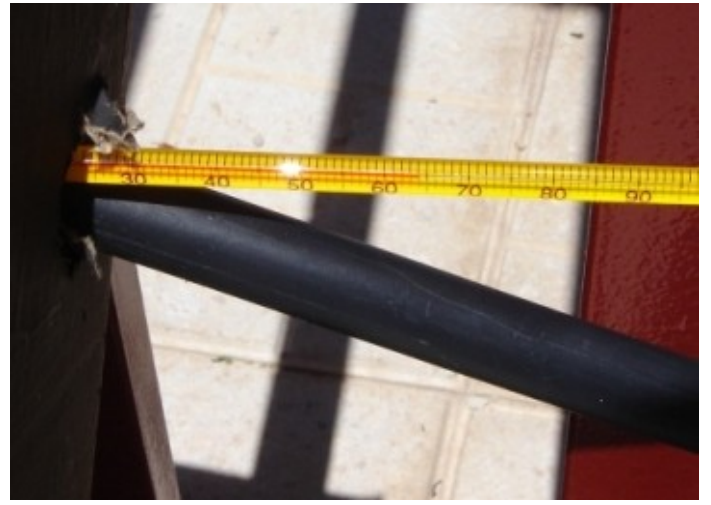

Figura 9

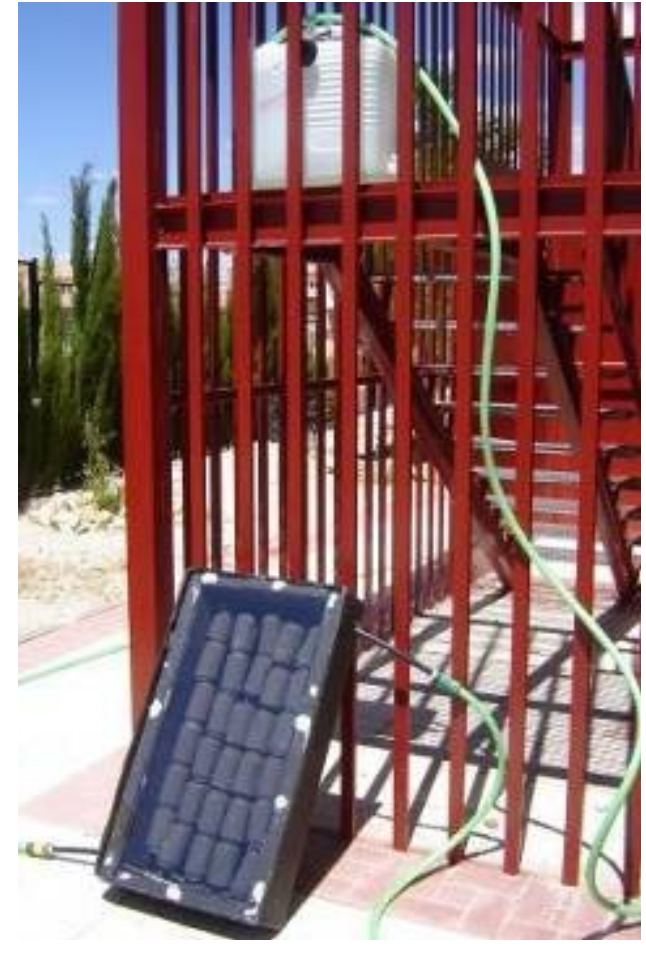

Figura 10 